\title{
MEDICAL IMAGE FUSION BASED ON NONSUBSAMPLED SHEARLET TRANSFORM AND IMPROVED SPIKING CORTICAL MODEL
}

\author{
Liu Shuaiqi ${ }^{\mathrm{a}, \mathrm{b}}$, Zhang Tao ${ }^{\mathrm{c}}$, Zhao Jie ${ }^{\mathrm{a}, \mathrm{b}}$, Li Huiyaa, \\ and Wang Xuehu ${ }^{a, b}$ \\ ${ }^{a}$ College of Electronic and Information Engineering, Hebei University, Baoding \\ Hebei, P. R. China \\ ${ }^{b}$ Key Laboratory of Digital Medical Engineering of Hebei Province, Baoding \\ Hebei, P. R. China \\ 'TCM Hospital of Shijiazhuang City, Shijiazhuang Hebei, P. R. China
}

\begin{abstract}
Many fusion methods have been proposed to fuse medical image, but these approaches alway lead to fusion image distortion or image information loss. To overcome the above disadvantages, combined with nonsubsampled shearlet transform (NSST) and spiking cortical model (SCM), a new medical image fusion is proposed. First, NSST is utilized for decomposition of the source images. Secondly, the low and high frequency coefficients of NSST are all fused by fired map of improved SCM (ISCM), which motivated by larger summodified-Laplacian (SML). Finally, the fusion image is gained by inverse NSST. The algorithm can both preserve the information of the source images well and suppress pixel distortion. Experimental results demonstrate that the proposed method outperforms the state-of-the-art fusion methods.
\end{abstract}

${ }^{*}$ Corresponding author.

E-mail address: li_huiya@yahoo.com.cn (Li Huiya).

Copyright () 2015 Scientific Advances Publishers

2010 Mathematics Subject Classification: 80.

Submitted by Haitao Xu.

Received May 20, 2015 
Keywords: medical image fusion, nonsubsampled shearlet transform, spiking cortical model.

\section{Introduction}

There are various modalities of medical images today such as computed tomography (CT), magnetic resonance angiography (MRA), magnetic resonance imaging (MRI), and functional MRI (fMRI) etc. [1]. Different modality medical image reflects different information of human organs. Doctor may get great help from one fused image, which is fused by complimentary features of different imaging modalities $[2,3]$.

Up to now, a lot of medical image fusion algorithms have been proposed. Generally, medical image fusion can be divided into three levels: pixel level fusion, feature level fusion, and decision level fusion [1]. Image fusion method at pixel-level can be divided into two categories: spatial domain algorithms and transform domain algorithms. The spatial domain algorithms mainly include principal component analysis (PCA) [4], guided filtering based method (GFF) [5] and so on. The transform domain algorithms are mainly based on multiresolution geometric analysis (MGA) tool domain and they can be divided into local energy function (such as image fusion algorithm based on wavelet [6], contourlet (CT) [7]) and artificial neural network (ANN) (such as nonsubsampled contourlet transform (NSCT) with pulse couple neural network (PCNN) [8], ripplet with PCNN [9], shearlet with PCNN [10]) by different fused rules.

MGA tools mainly contain discrete wavelet transform (DWT), CT, NSCT, and shearlet (ST). They are all successfully applied to image fusion. However, CT is translational variability [7]. NSCT constructed by non-subsampled Laplacian transform and non-subsampled directional filter can overcome this disadvantage, and NSCT-based image fusion method arrive satisfactory fusion performance. However, the high computational complexity of NSCT cause it is difficult to be applied to real time applications. Unlike to NSCT, ST [11-14] has a rigorous mathematical framework and smaller support sizes of shear filter can 
accelerate the computation speed. But ST also lack shift-variance and this will lead some artifacts when fused by using ST. NSST is proposed to overcome the disadvantages of NSCT an ST [11]. Compared with current MGA tools, NSST is not only shift-invariance, but also has better sparse representation ability and much faster computational speed. So, image fusion based on NSST can achieve better fused performance.

However, MGA tools fusion methods also lead to pixel distortion due to nonlinear operations in the transform domain $[9,10]$. On the contrary, much less distortions are introduced if linear fusion rules in the spatial domain or ANN [15]. PCNN is a simplified model, which is suited to realizing synchronous pulse excitation behaviour. This model has been efficiently applied to image processing in applications such as image segmentation, image fusion, etc. However, the parameters of PCNN are too many and it is computation complexity and time consuming. SCM is one of the simplified PCNN models that is mainly derived from Eckhorn's model and deduced from primate visual cortex, and also has been proved an effective image processing tool [16]. Image fusion based on SCM also can archived good effect, such as just medical image fusion motivated by source images gray values in [17], multisource image fusion using spatial frequency and SCM based on DWT in [18], multimodal medical image fusion based on SCM in [19], and multifocus image fusion based on SCM in [20]. Though in these image fusion methods, the fired maps of SCM motivated by source images can perform better effect than PCNN, the synaptic linking weight of SCM is const. In our opinion, the synaptic linking weight should be related to the clarity of source images. So, we give an improved SCM (ISCM) with adaptive synaptic linking weight. ISCM perform better than SCM in medical image fusion by using image fusion method in [17], see Figure 4.

Considering the advantages of MGA tools and SCM, we can easily get the conclusion as follows. First, NSST has significant superiorities than other MGA tools and NSST can also be applied to more research fields. Second, ISCM can effectively suppress the MGA fusion method's image distortion. As a result, we attempt to combine the advantages of both NSST and SCM together, and propose a novel medical image fusion method based on NSST and ISCM. 
This paper is organized as follows. In Section 2, we give a brief review of NSST theory. In Section 3, we introduce the working principle of SCM and its improvement. In Section 4, we give the steps of the new image fusion algorithm based on NSST and SCM. In Section 5, we demonstrate the experimental results of the proposed and the comparisons with other typical fusion methods. And in the last section, we explore some conclusions.

\section{Non-Subsampled Shearlet Transform}

ST is a new multi-scale geometric analysis algorithm which inherits advantages of contourlet and curvelet transform [11]. ST theory is based on composite wavelets. In dimension $n=2$, the affine systems with composite dilations are the collections of the form [11, 12]:

$$
\Omega_{\boldsymbol{A} \boldsymbol{B}}(\psi)=\left\{\begin{array}{l}
\psi_{j, l, \mathbf{k}}(\mathbf{x})=|\operatorname{det} \boldsymbol{A}|^{j / 2} \psi\left(\boldsymbol{B}^{l} \boldsymbol{A}^{j} \mathbf{x}-\mathbf{k}\right) \\
: j, l \in Z, \mathbf{k} \in Z^{2}
\end{array}\right\},
$$

where $\psi \in L^{2}\left(R^{2}\right), \boldsymbol{A}, \boldsymbol{B}$ are $2 \times 2$ invertible matrices and $|\operatorname{det} \boldsymbol{B}|=1$.

When for any $f \in R^{2}, \Omega_{A B}(\psi)$ forms a Parseval frames (also called tight frame), we call the elements of $\Omega_{A B}(\psi)$ composite wavelet. The dilations $\boldsymbol{A}^{j}$ are associated with scale transformations, while the matrices $\boldsymbol{B}^{l}$ are associated to area-preserving geometrical transform, such as rotations and shear [11-14]. Normally, $\boldsymbol{A}=\boldsymbol{A}_{0}=\left(\begin{array}{ll}4 & 0 \\ 0 & 2\end{array}\right)$ is the anisotropic dilation matrix, and $\boldsymbol{B}=\boldsymbol{B}_{0}=\left(\begin{array}{ll}1 & 1 \\ 0 & 1\end{array}\right)$ is shear matrix in (1), then, we can construct a tiling of the frequency like Figure 1. 


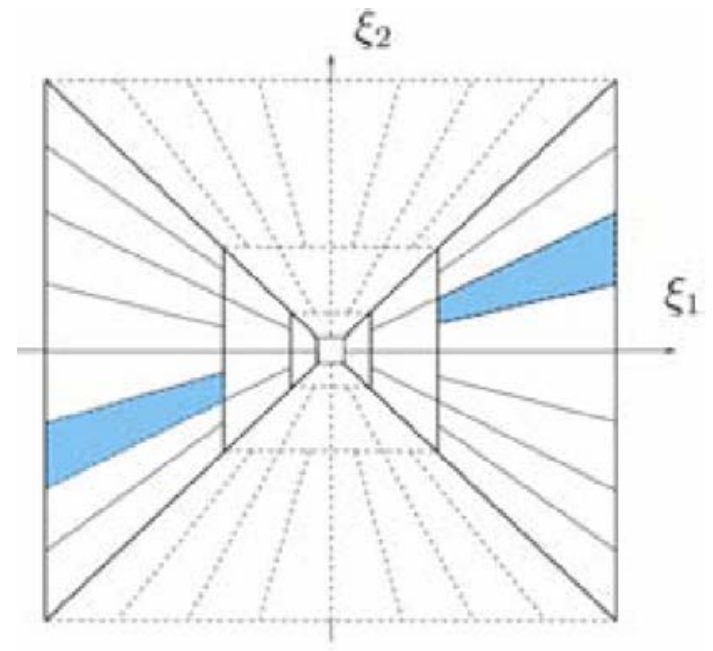

Figure 1. The tiling of the frequency plane induced by the ST.

Let $\psi_{j, l, \mathbf{k}}^{(\theta)}(\mathbf{x})=2^{\frac{3 j}{2}} \psi^{(\theta)}\left(\boldsymbol{B}_{1}^{l} \boldsymbol{A}_{1}^{j} \mathbf{x}-k\right)$. That is, each element $\hat{\psi}_{j, l, \mathbf{k}}$ is supported on a pair of trapezoids, of approximate size $2^{2 j} \times 2^{j}$, oriented along lines of slope $l 2^{-j}$, see Figure 2 .

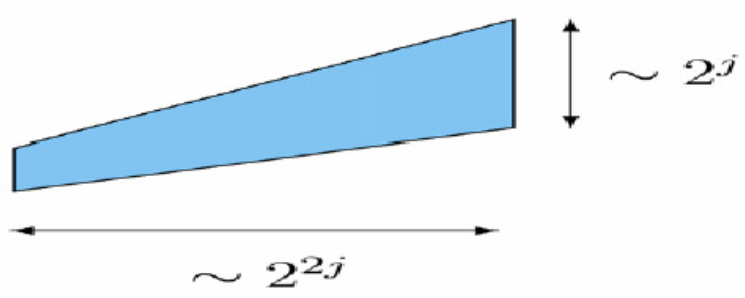

Figure 2. The frequency support of ST.

If $f \in L^{2}\left(R^{2}\right)$, then its continuous ST is defined as:

$$
S H_{\psi}=\left\langle f, \psi_{j, l, \mathbf{k}}^{(\theta)}\right\rangle,
$$

where $j \geq 0, l=-2^{j}, 2^{j}-1, \mathbf{k} \in Z^{2}, \theta=0,1$. 
The discretization process of NSST [11] is composed of two phases: multi-scale factorization and multi-orientation factorization. Nonsubsampled pyramidis utilized to complete multi-scale factorization, which can produce $(k+1)$ sub-images which consist of one low frequency image and $k$ high frequency images whose sizes are all the same as the source image, where $k$ denotes the number of decomposition levels [11].

\section{Spiking Cortical Model}

The SCM proposed by Zhan et al. [16] is derived from Eckhorn's model and it conforms to the physiological characteristic of human visual neural system. Indeed, Wang's method [17] provides an effective means for fusion of the different kinds of medical images. In the spiking cortical model, each neuron consists of three parts: feeding and linking field, modulating product, and pulse generator, see Figure 3.

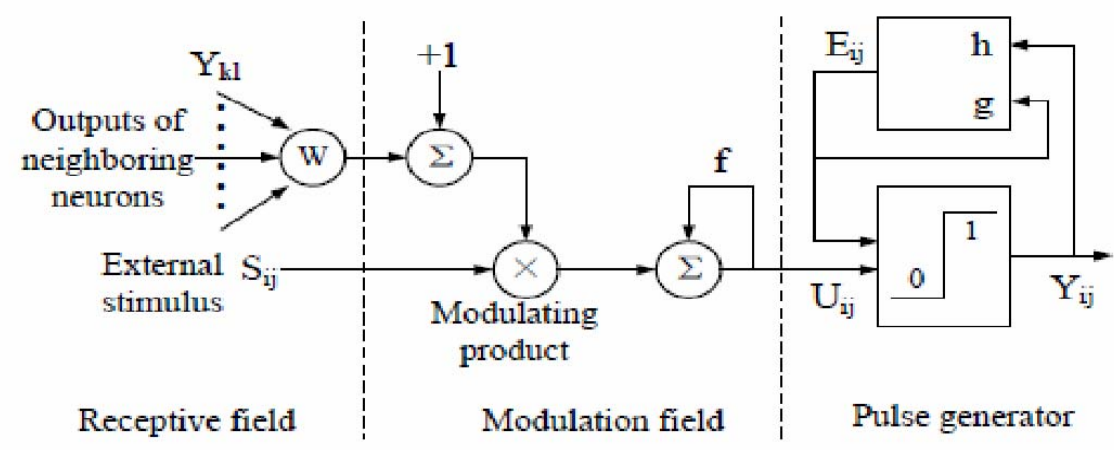

Figure 3. SCM model.

In the following expressions, the indexes $i$ and $j$ refer to the pixel location in the image, $k$ and $l$ refer to the location of its neighbouring pixels and $n$ denotes the current iteration times. The receiving and linking field and modulating product is given by

$$
U_{i j}(n)=f U_{i j}(n-1)+S_{i j} \sum_{k l} W_{i j k l} Y_{k l}(n-1)+S_{i j}
$$


where $U_{i, j}(n)$ is the internal activity and $f$ is the attenuation coefficient of $U_{i, j}(n) ; S_{i, j}$ is the external stimulus; $W_{i j k l}$ is the synaptic linking weight and $Y_{i j}(n-1)$ is the previous output pulse.

The pulse generator determines the firing events in the model in (4). $Y_{i j}$ depends on the internal activity and threshold.

$$
Y_{i j}(n)= \begin{cases}1, & \text { if } 1 /\left(1+\exp \left(U_{i j}(n)-E_{i j}(n)\right)\right)>0.5 \\ 0, & \text { otherwise. }\end{cases}
$$

The dynamic threshold of the neuron is defined as

$$
E_{i j}(n)=g E_{i j}(n-1)+h Y_{i j}(n-1)
$$

where $g$ denotes attenuation coefficient, and $h$ denotes the threshold magnitude coefficient. And the fire number can be represents as follow:

$$
T_{i j}(n)=T_{i j}(n-1)+Y_{i j}(n),
$$

where $T_{i j}(n)$ denotes the total number of the fired times of neurons after the current iteration.

From above definition, we can find the synaptic linking weight of SCM is const. In our opinion, the synaptic linking weight should be related to the clarity of source images. An adaptive synaptic linking weight is defined by using SML of the source images. That is,

$$
W_{i j}=S M L(I(i, j)) \text {. }
$$

Without losing of generality, we suppose that $I$ is a medical image, and external stimulus $S_{i, j}$ also can be represented by SML of the source images. That is,

$$
S_{i j}=S M L(I(i, j))
$$


The SML at the pixel $(i, j)$ is defined as follows [15]:

$$
\operatorname{SML}(I(i, j))=\sum_{(x, y) \in \omega_{(i, j)}}[M L(I(x, y))]^{2}
$$

where $\omega_{(i, j)}$ denotes a rectangular window centered at the pixel $(i, j)$, for $\forall(x, y) \in \omega_{(i, j)}$, we have the follow definition:

$$
\begin{aligned}
M L(I(x, y))= & \mid 2 I(x, y)-I(x-\text { step }, y)-I(x+\text { step, } y) \mid \\
& +\mid 2 I(x, y)-I(x, y-\text { step })-I(x, y+\text { step }) \mid,
\end{aligned}
$$

where step denotes a variable spacing between pixels. Generally, step equals to 1 .

To show the effect of new synaptic linking weight of SCM, the fusion image by SCM and ISCM with external stimulus $S_{i, j}$ both are source images is shown in Figure 4. Obviously, Figure 4(b) is clearer than Figure 4(a), and it prove that adaptive synaptic linking weight can suppress effectively artificial texture and reserve more details of medical source images.

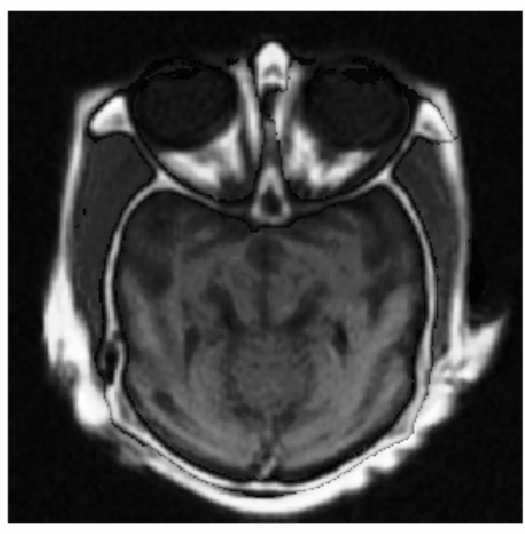

(a)

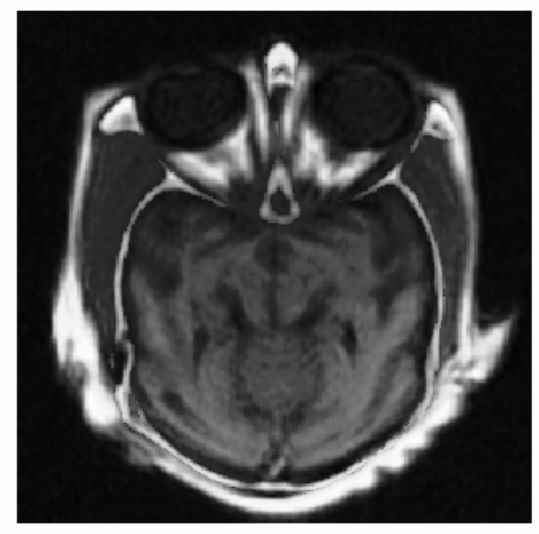

(b)

Figure 4. The performance of SCM and ISCM, (a) the fused image based on SCM, (b) the fused image based on ISCM. 


\section{Image Fusion Based on NSST and ISCM}

The proposed image fusion algorithm (NSST-ISCM) is described as follows. Without losing of generality, we suppose that $A$ and $B$ are two medical images with different sensor to fuse, $F$ is fused image.

First, we utilize NSST to decompose image $A$ and $B$, with decomposition coefficients $C_{A}^{l, \theta}(i, j)$ and $C_{B}^{l, \theta}(i, j)$, where $l$ and $\theta$ denote the scale and direction of decomposition, respectively. When $l$ is zero, they denote low frequency coefficients, and otherwise they denote high frequency coefficients. $(i, j)$ denotes the position of pixel.

Secondly, SML of the coefficients of NSST is computed by Equation (9). $S M L_{A}^{l, \theta}$ denotes SML of $C_{A}^{l, \theta}(i, j)$ and $S M L_{B}^{l, \theta}$ denotes SML of $C_{B}^{l, \theta}(i, j)$. The SML of the NSST coefficients are normalized and taken as the stimuli of the two ISCMs, respectively.

$$
T_{A}=\operatorname{ISCM}\left(S M L_{A}^{l, \theta}\right), \quad T_{B}=\operatorname{ISCM}\left(S M L_{B}^{l, \theta}\right),
$$

where $I S C M$ () denotes the ISCM with synaptic linking weight. $T_{A}$ and $T_{B}$ are the total firing times motivated by $S M L_{A}^{l, \theta}$ and $S M L_{B}^{l, \theta} \cdot C_{F}^{l, \theta}(i, j)$ are the fused NSST coefficients.

$$
C_{F}^{l, \theta}(i, j)= \begin{cases}C_{A}^{l, \theta}(i, j), & \text { if } T_{A}-T_{B}>T t h, \\ \left(C_{A}^{l, \theta}(i, j)+C_{B}^{l, \theta}(i, j)\right) / 2, & \text { if }\left|T_{A}-T_{B}\right|=T t h, \\ C_{B}^{l, \theta}(i, j), & \text { otherwise }\end{cases}
$$

where $T t h=0.5$ denotes the constant threshold. Basic fused image $F_{b}$ can be got by inverse NSST. 


\section{Experimental Results}

In order to evaluate the performance of the proposed fusion method, we introduce some objective criteria such as mutual information (MI) [21], $\mathrm{Q}^{\mathrm{AB} / \mathrm{F}}$ metric [21], $\mathrm{L}^{\mathrm{AB} / \mathrm{F}}$ metric [21], and $\mathrm{N}^{\mathrm{AB} / \mathrm{F}}$ metric [21]. MI measures the amount of information transferred to the fused image from the source images. The $\mathrm{Q}^{\mathrm{AB} / \mathrm{F}}$ utilizes Sobel edge detector to measure the amount of edge information, which is transferred from the source images to the fused image. The higher $\mathrm{MI}$ and $\mathrm{Q}^{\mathrm{AB} / \mathrm{F}}$, and the better fusion performance will be. $\mathrm{L}^{\mathrm{AB} / \mathrm{F}}$ is introduced to evaluate the information lost during the fusion process. $\mathrm{N}^{\mathrm{AB} / \mathrm{F}}$ represents fusion artifacts that introduced into the fused image in fusion process. It is clear that the $\mathrm{L}^{\mathrm{AB} / \mathrm{F}}$ and $\mathrm{N}^{\mathrm{AB} / \mathrm{F}}$ are smaller, the better the result is. It should point that the sum of $\mathrm{Q}^{\mathrm{AB} / \mathrm{F}}, \mathrm{L}^{\mathrm{AB} / \mathrm{F}}$, and $\mathrm{N}^{\mathrm{AB} / \mathrm{F}}$ should result in unity [21]. Further, the fused algorithms are evaluated by using the Matlab codes on Intel Core $22.6 \mathrm{GHz}$ machines with a $4 \mathrm{~GB}$ RAM.

To evaluate the performance of the proposed fusion method, the experiments have been performed on four pairs of multimodal medical images as shown in Figure 5. These pairs of images are divided into the four groups. Group a contain Figure 5(a) and (f), and they are the CT images shown the bones information and MRI image displayed the soft tissue, respectively. Group b contain Figure 5(b) and (g), and Figure 5(b) is B ultrasound image of thyroid tumor, as the anatomical imaging, can provide organ organization structure information. Figure 5(g) is SPECT image of thyroid tumors, as functional imaging, which can provide information of benign and malignant of thyroid tumor. Group c contain Figure 5(c) and (h), and they are CT image and T1-weighted MR-GAD image of several focal lesion s involving basal ganglia. Group d contain Figure 5(d) and (i), and they are T1-MRI and T2-MRI that involved the lesion in the frontal lobe. 


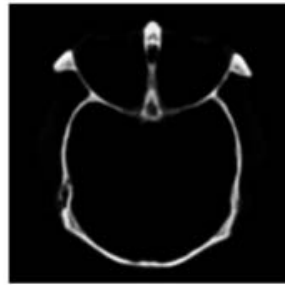

(a)

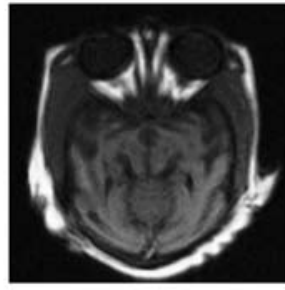

(f)

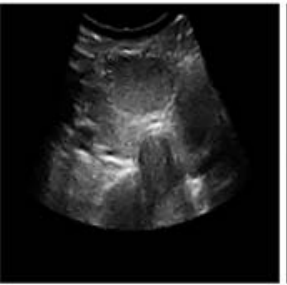

(b)

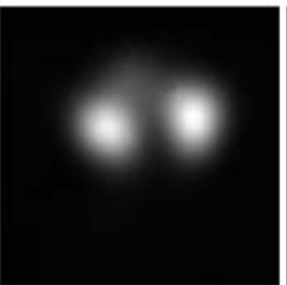

(g)

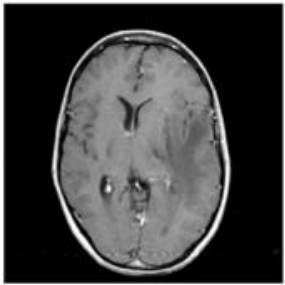

(c)

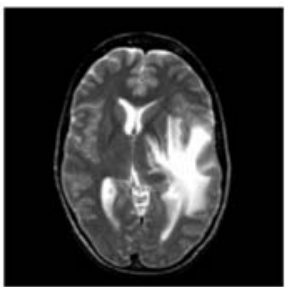

(h)

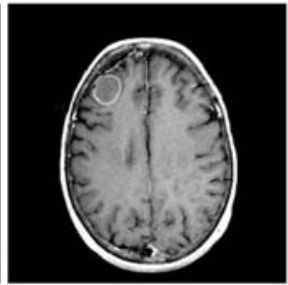

(d)

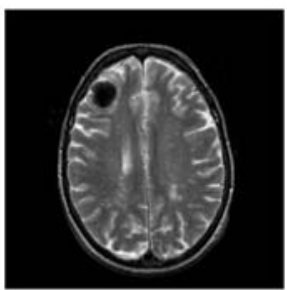

(i)

Figure 5. Different medical images to fuse.

The following algorithms are used for comparison studies in the experiments. (1) GFF based on guided filtering proposed in [5] (GFF). (2) Medical image fusion based on nonsubsampled direction complex wavelet transform proposed in [7] (NDCWT). (3) NSCT-based multimodal medical image fusion using $\mathrm{PCNN}$ and modified spatial frequency proposed in [8] (NSCT-SF-PCNN). (4) Fusion algorithm based on ST and PCNN proposed in [10] (ST-PCNN). (5) Fusion algorithm based on NSCT and SCM proposed in [18] (NSCT-SCM). For the sake of fair comparison, we used the parameters that were reported by the authors to yield the best fusion results. The parameters except $W_{i j k l}$ of our method are the same to fused method based on SCM in [17]. The decomposition level of NSST is 2, with 2, 32 directional. 


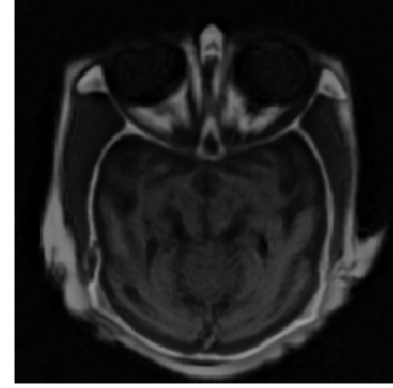

(a)

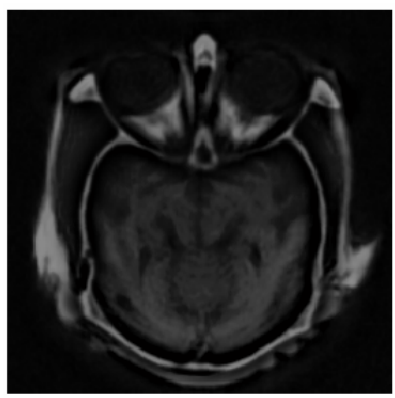

(d)

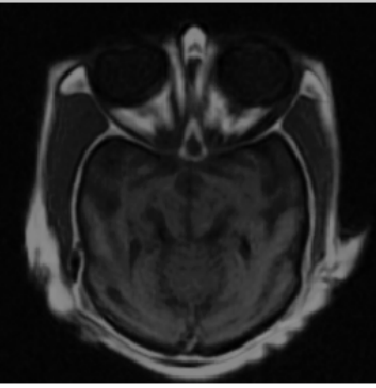

(b)

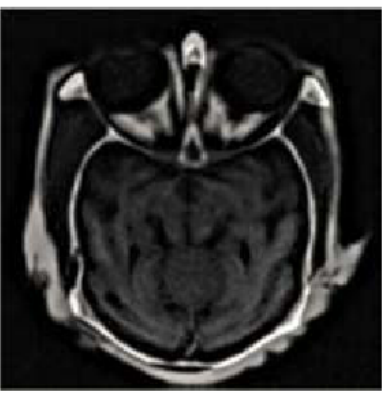

(e)

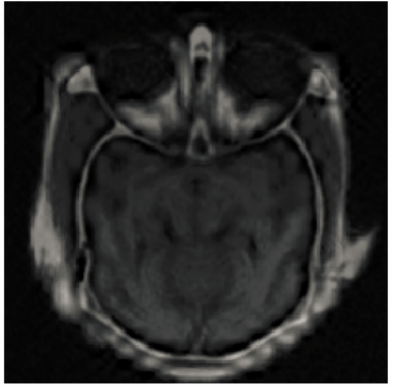

(c)

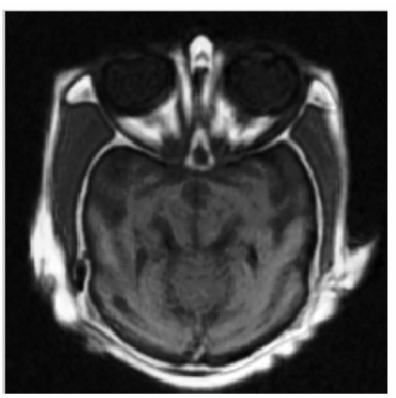

(f)

Figure 6. Fusion results of group a. (a)-(f) are the fusion image by GFF, NDCWT, NSCT-SF-PCNN, ST-PCNN, NSCT-SCM, and NSST-ISCM.

The above methods are utilized to fuse four group images in Figure 5, respectively. Figures 6-9 shows fused images by six fused methods. From the fusion results in Figure 6(a)-(f), it is easy to find that there are blocking effect and unwanted image degradations in Figure 6(c) and (d). It can be clearly seen that image fused by our method reach a higher contrast in all the fused methods. Comparing the fused image of each algorithm, we can see the proposed fusion algorithm preserve the texture information of source images well. At the same time, less useless image information such as block effect and artifacts are introduced in the fusion images in the present scheme.

The same to Figure 6, from the fusion results of the six algorithms in Figure 7(a)-(f), our method has best visual effect in all the fused methods. Figure 7(a)-(c) show that GFF, NDCWT, NSCT-SF-PCNN, and ST-PCNN cannot fuse this kind medical images well. And Figure 7(e)-(f) show that 
method based on SCM can achieve much better performances. Comparing the fused image of SCM, our method preserves more texture information of source images, which should be attributed to ISCM.

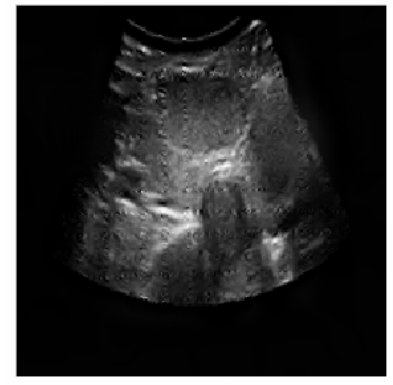

(a)

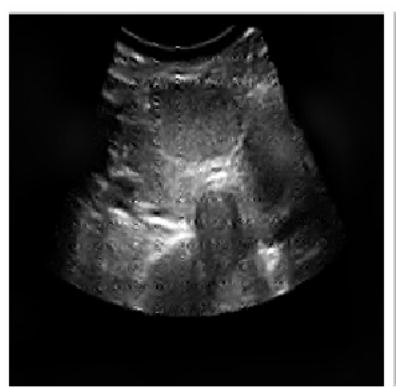

(d)

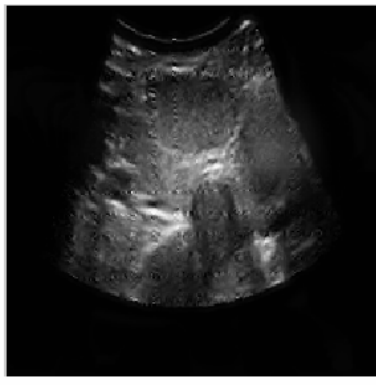

(b)

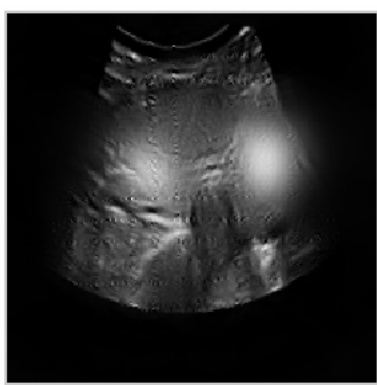

(e)

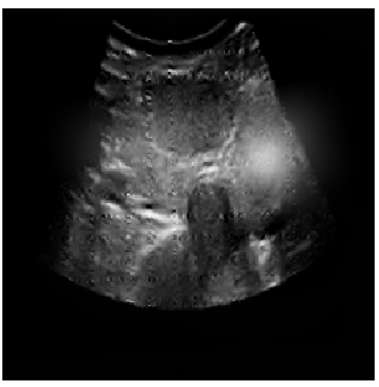

(c)

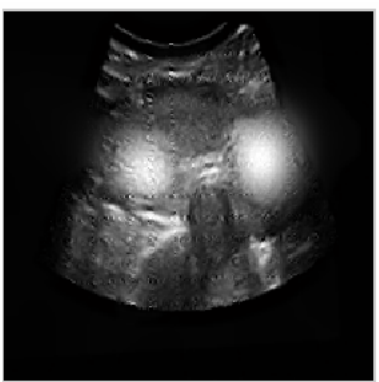

(f)

Figure 7. Fusion results of group b. (a)-(f) are the fusion image by GFF, NDCWT, NSCT-SF-PCNN, ST-PCNN, NSCT-SCM, and NSST-SCM.

From the objective criteria shown in Table 1, our algorithm has the best objective criteria. The highest $\mathrm{MI}$ and $\mathrm{Q}^{\mathrm{AB} / \mathrm{F}}$ mean that most useful information and edge information converted into the fused result by our algorithm. The least $\mathrm{L}^{\mathrm{AB} / \mathrm{F}}$ means that least information of source images is lost by our method. The least $\mathrm{N}^{\mathrm{AB} / \mathrm{F}}$ means that least fusion artifacts are introduced into the fused image by our method. So our method can be regarded as a kind of good medical image fusion algorithm. 
Table 1. Objective criteria by each fused method in the fusion of Figure 6 and Figure 7

\begin{tabular}{l|cccc|cccc}
\hline & \multicolumn{4}{|c|}{ Group a } & \multicolumn{4}{c}{ Group b } \\
\hline $\begin{array}{l}\text { Fusion } \\
\text { method }\end{array}$ & MI & $\mathrm{Q}^{\mathrm{AB} / \mathrm{F}}$ & $\mathrm{L}^{\mathrm{AB} / \mathrm{F}}$ & $\mathrm{N}^{\mathrm{AB} / \mathrm{F}}$ & $\mathrm{MI}$ & $\mathrm{Q}^{\mathrm{AB} / \mathrm{F}}$ & $\mathrm{L}^{\mathrm{AB} / \mathrm{F}}$ & $\mathrm{N}^{\mathrm{AB} / \mathrm{F}}$ \\
\hline GFF & 3.4313 & 0.7789 & 0.1405 & 0.0806 & 3.4736 & 0.7649 & 0.1698 & 0.0653 \\
NDCWT & 4.3905 & 0.7555 & 0.1401 & 0.1038 & 3.3573 & 0.7424 & 0.1759 & 0.0781 \\
$\begin{array}{l}\text { NSCT-SF- } \\
\text { PCNN }\end{array}$ & 4.8300 & 0.3635 & 0.1480 & 0.4885 & 3.1594 & 0.7073 & 0.1875 & 0.1052 \\
$\begin{array}{l}\text { ST- } \\
\text { PCNN }\end{array}$ & 2.2828 & 0.6761 & 0.2175 & 0.1064 & 4.8966 & 0.7627 & 0.1854 & 0.0519 \\
$\begin{array}{l}\text { NSCT- } \\
\text { SCM }\end{array}$ & 3.5955 & 0.8037 & 0.1350 & 0.0614 & 3.2116 & 0.7389 & 0.1771 & 0.0840 \\
$\begin{array}{l}\text { NSST- } \\
\text { SCM }\end{array}$ & $\mathbf{4 . 9 2 4 2}$ & $\mathbf{0 . 8 6 8 3}$ & $\mathbf{0 . 1 2 5 3}$ & $\mathbf{0 . 0 0 6 4}$ & $\mathbf{4 . 9 0 7 0}$ & $\mathbf{0 . 8 8 5 7}$ & $\mathbf{0 . 0 8 2 1}$ & $\mathbf{0 . 0 3 2 2}$ \\
\hline
\end{tabular}

Figure 8 and Figure 9 show fused images of group c and d by six fused methods. The same to Figure 6 and Figure 7, from the fusion results in Figure 8(a)-(f) and Figure 9(a)-(f), our method both has a higher contrast in all the fused methods and preserve the texture information of source images, suppress useless image information such as block effect and artifacts.

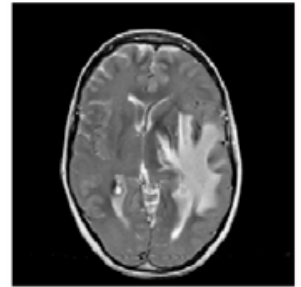

(a)

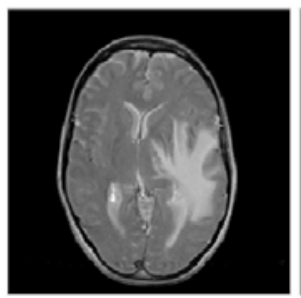

(d)

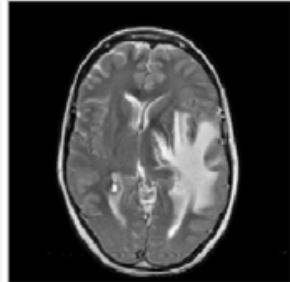

(b)

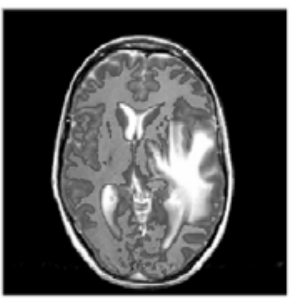

(e)

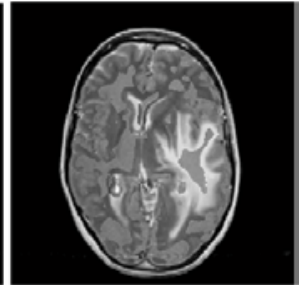

(c)

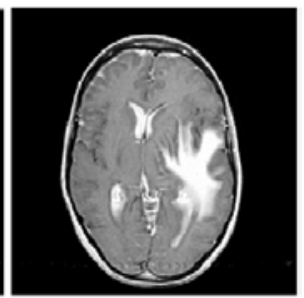

(f)

Figure 8. Fusion results of group c. (a)-(f) are the fusion image by GFF, NDCWT, NSCT-SF-PCNN, ST-PCNN, NSCT-SCM, and NSST-SCM. 


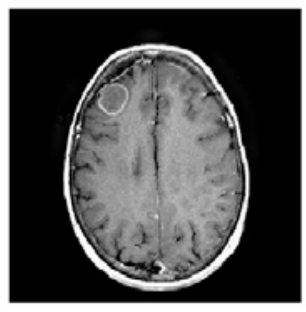

(a)

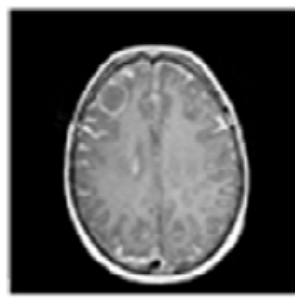

(d)

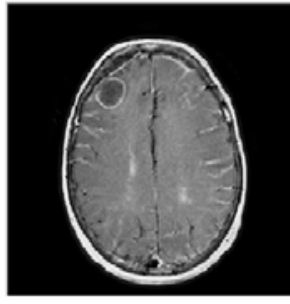

(b)

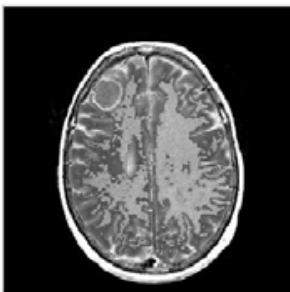

(e)

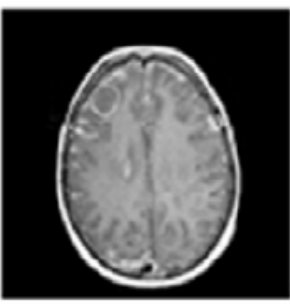

(c)

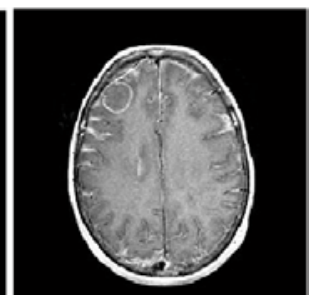

(f)

Figure 9. Fusion results of group d. (a)-(f) are the fusion image by GFF, NDCWT, NSCT-SF-PCNN, ST-PCNN, NSCT-SCM, and NSST-SML.

From the objective criteria shown in Table 2, our algorithm always has the best objective criteria. So our method can be regarded as a robust medical image fusion algorithm.

Table 2. Objective criteria by each fused method in the fusion of Figure 8 and Figure 9

\begin{tabular}{|c|c|c|c|c|c|c|c|c|}
\hline \multirow[b]{2}{*}{$\begin{array}{l}\text { Fusion } \\
\text { method }\end{array}$} & \multicolumn{4}{|c|}{ Group c } & \multicolumn{4}{|c|}{ Group d } \\
\hline & MI & $\mathrm{Q}^{\mathrm{AB} / \mathrm{F}}$ & $\mathrm{L}^{\mathrm{AB} / \mathrm{F}}$ & $\mathrm{N}^{\mathrm{AB} / \mathrm{F}}$ & MI & $\mathrm{Q}^{\mathrm{AB} / \mathrm{F}}$ & $\mathrm{L}^{\mathrm{AB} / \mathrm{F}}$ & $\mathrm{N}^{\mathrm{AB} / \mathrm{F}}$ \\
\hline GFF & 3.4548 & 0.8217 & 0.1458 & 0.0325 & 3.5974 & 0.7625 & 0.2089 & 0.0286 \\
\hline NDCWT & 3.4854 & 0.7037 & 0.1973 & 0.0990 & 3.2677 & 0.5641 & 0.2561 & 0.1798 \\
\hline $\begin{array}{l}\text { NSCT-SF- } \\
\text { PCNN }\end{array}$ & 3.4376 & 0.7486 & 0.2236 & 0.0278 & 3.5588 & 0.5710 & 0.2874 & 0.1416 \\
\hline ST-PCNN & 3.3719 & 0.7157 & 0.2354 & 0.0489 & 3.1847 & 0.7040 & 0.2290 & 0.0670 \\
\hline $\begin{array}{l}\text { NSCT- } \\
\text { SCM }\end{array}$ & 3.2889 & 0.8060 & 0.1577 & 0.0363 & 3.1797 & 0.7579 & 0.2028 & 0.0393 \\
\hline $\begin{array}{l}\text { NSST- } \\
\text { SCM }\end{array}$ & 3.7394 & 0.8334 & 0.1435 & 0.0231 & 3.6185 & 0.7796 & 0.1951 & 0.0252 \\
\hline
\end{tabular}




\section{Conclusion}

A new fused method based on NSST and improved SCM is proposed to improve the fusion effect. ISCM preserves more texture information of source images and robust than PCNN. The new fused method can extend SCM to more widely using in other kinds medical images fusion. The experimental results show that NSST has significant superiorities than other MGA tools in medical fusion, and ISCM can effectively suppress the MGA fusion method's image distortion. So, the proposed method is better than state-of-the-art medical image fusion methods both visual appearance and objective criteria.

\section{Acknowledgements}

This work was supported in part by Natural Science Foundation of China under grant 61401308, Natural Science Foundation of Hebei Province under grant 2013210094, Natural Science Foundation of Hebei University under grant 2014-303, Science and Technology Research and Development Project of Baoding City under grant 14ZG036, 15ZG016, and Hebei University Laboratory Development Project under grant sy2015009, sy2015057. Sources images can be downloaded on http://www.med.harvard.edu/aanlib/home.html and Hebei University.

\section{References}

[1] K. S. Tamilselvan and G. Murugesan, Survey and analysis of various image fusion techniques for clinical CT and MRI images, International Journal of Imaging Systems and Technology 24(2) (2014), 193-202.

[2] Y. M. Zhu and S. M. Cochoff, An object-oriented framework for medical image registration, fusion, and visualization, Computer Methods and Programs in Biomedicine 82(3) (2006), 258-267.

[3] L. Wang, B. Li and L. F. Tian, Multi-modal medical image fusion using the interscale and intra-scale dependencies between image shift-invariant shearlet coefficients, Information Fusion 19 (2014), 20-28.

[4] U. Patil and U. Mudengudi, Image fusion using hierarchical PCA [C]. 2011 International Conference on image Information Processing (ICIIP), Nov. 2011, IEEE Press, pp. 1-6. 
[5] S. T. Li, X. D. Kang and J. W. Hu, Image fusion with guided filtering, IEEE transactions on Image Processing 22(7) (2013), 2864-2875.

[6] X. Zhang, Y. Zheng and Y. Peng et al., Research on multi-mode medical image fusion algorithm based on wavelet transform and the edge characteristics of images, 2nd International Congress on Image and Signal Processing, IEEE Press (2009), 1-4.

[7] S. Q. Liu, J. Zhao and P. Geng et al., Medical image fusion based on nonsubsampled direction complex wavelet transform, International Journal of Applied Mathematics and Machine Learning 1(1) (2014), 21-34.

[8] S. Das and M. K. Kundu, NSCT-based multimodal medical image fusion using pulsecoupled neural network and modified spatial frequency, Medical and Biological Engineering and Computing 50(10) (2012), 1105-1114.

[9] S. Das and M. K. Kundu, Ripplet based multimodality medical image fusion using pulse-coupled neural network and modified spatial frequency, 2011 International Conference on Recent Trends in Information Systems (ReTIS), IEEE Press, Dec. 2011, pp. 229-234.

[10] P. Geng, Z. Wang and Z. Zhang et al., Image fusion by pulse couple neural network with shearlet, Optical Engineering 51(6) (2012), 067005-1-067005-7.

[11] G. Easley, D, Labate and W. Q. Lim, Sparse directional image representation using the discrete shearletstransform, Applied and Computational Harmonic Analysis 25(1) (2008), 25-46.

[12] G. Kutyniok, J. Lemvig and W. Q. Lim, Compactly supported shearlets are optimally sparse, Journal of Approximation Theory 163(11) (2011), 1564-1589.

[13] W. Q. Lim, The discrete shearlets transform: A new directional transform and compactly supported shearlets frames, IEEE Trans. Image Proc. 19(5) (2010), 1166-1180.

[14] S. Q. Liu, S. H, Hu and Y. Xiao, et al. Bayesian shearlet shrinkage for SAR image denoising via sparse representation, Multidimensional Systems and Signal Processing 25(4) (2014), 683-701.

[15] D. Guo, J. Yan and X. Qu, High quality multi-focus image fusion using self-similarity and depth information, Optics Communications 338(1) (2015), 138-144.

[16] K. Zhan, H. Zhang and Y. Ma, New spiking cortical model for invariant texture retrieval and image processing, IEEE Transactions on Neural Networks 20(12) (2009), 1980-1986.

[17] R. Wang, Y. Wu and M. Ding et al., Medical image fusion based on spiking cortical model, SPIE Medical Imaging, International Society for Optics and Photonics (2013) 867610-867610-7.

[18] N. Wang, Y. Ma and W. Wang, DWT-based multisource image fusion using spatial frequency and simplified pulse coupled neural network, Journal of Multimedia 9(1) (2014), 159-165. 
[19] N. Wang, Y. Ma and K. Zhan et al., Multimodal medical image fusion framework based on simplified PCNN in nonsubsampled contourlet transform domain, Journal of Multimedia 8(3) (2013), 270-276.

[20] N. Wang, Y. Ma and K. Zhan, Spiking cortical model for multifocus image fusion, Neurocomputing 130 (2014), 44-51.

[21] C. S. Xydeas and V. Petrovic, Objective image fusion performance measure, Electronics Letters 36(4) (2000), 308-309. 